\title{
Validity and Reliability of the WHOQOL-BREF in the measurement of the quality of life of Sickle Disease Patients in Bahrain
}

Amer Almarabheh ( $\square$ amerjka@agu.edu.bh )

Arabian Gulf University https://orcid.org/0000-0002-4279-2371

Manal Al Ghamdi

Arabian Gulf University

Abdulla Elbarbary

Arabian Gulf University

Ahmed Alqashar

Arabian Gulf University

Faisal alserdieh

Arabian Gulf University

Fatema Alahmed

Arabian Gulf University

Hasan Alhaddar

Arabian Gulf University

Lulwa AlSada

Arabian Gulf University

Mohamed yosri

Arabian Gulf University

Mahmood Omran

Arabian Gulf University

Mina Khudhair

Arabian Gulf University

Motasem Salih

Arabian Gulf University

Noora fuad

Arabian Gulf University

sadok chlif

Arabian Gulf University

Afif Ben Salah

Arabian Gulf University 
Research

Keywords: WHOQOL-BREF, Validity, Reliability, Quality of Life, Sickle cell disease

Posted Date: September 17th, 2021

DOI: https://doi.org/10.21203/rs.3.rs-892568/v1

License: (c) (i) This work is licensed under a Creative Commons Attribution 4.0 International License. Read Full License 


\section{Abstract \\ Background}

Limited attention is devoted to the improvement of the quality of life of patients suffering from the negative consequences of Sickle cell disease (SCD). Our study focuses on the evaluation of the performance of the WHOQOL-BREF as a tool to measure the quality of life of SCD Patients in Bahrain.

\section{Methods}

We conducted a cross-sectional study that enrolled 273 SCD patients selected using a simple random sampling technique from primary health-care centers in Bahrain in 2019. A designed questionnaire including the WHOQOL-BREF was filled by the patients during face-to-face interviews in the health centers. The reliability of the WHOQOL-BREF was assessed by standardized Cronbach's alpha coefficient, and the validity was measured by convergent validity, principal component analysis and confirmatory factor analysis.

\section{Results}

The WHOQOL-BREF had good internal consistency as Cronbach's alpha coefficient for the overall scale was 0.91 . The convergent validity results indicated that the correlation coefficients values for all scale domains are significantly correlated at $a<0.01$. Confirmatory factor analysis found that the four-domain structure produced a robust fit to the data.

\section{Conclusions}

The WHOQOL-BREF tool has high internal consistency and validity in assessing the quality of life of Sickle Disease patients in Bahrain.

\section{Background}

Sickle cell disease (SCD) is an inherited autosomal recessive blood disorder that affects the structure of hemoglobin and is characterized by sickling of the red blood cells (RBCs). It is a life- threatening condition that accounts for important morbidity load among children and adults worldwide $[1,2]$ SCD is most common in Sub-Saharan Africa, the Caribbean, India, the Middle East, and the Mediterranean basin [3]. However, due to migration, it expanded as a significant health problem in Europe and the United States [4]. Pain crisis of SCD and is the main cause of hospitalization, mortality and might affect the quality of life of SCD patients. 
The concept of quality of life (QoL) is widely used in a highly diverse range of disciplines and contexts. Sociologists, physicians, psychologists, and economists are among those using this concept routinely, with the main aims to improve the living conditions of individuals and societies [5]. The evaluation of Quality of life (Qol), as part of mental health, is nowadays considered as fundamental in all medical specialties [6]. It has been recommended in routine practice [7], and in evaluating mental health services [8]. The WHOQOL-BREF is a tool developed by the World Health Organization to evaluate QoL in various areas of health care and different cultural settings, languages, and countries $[9,10]$. It is a 26 item selfreport instrument derived from the original 100-item instrument, which had demonstrated reasonable validity and reliability. The WHOQOL-BREF evaluates four dimensions of quality of life ("Physical Health", "Psychological Health", "Social Relationships", "Environment"), and contains two items that are examined separately (an individual's "overall perception of quality of life" and an individual's "overall perception of health") $[10,11]$. The reliability and validity of WHOQOL-BREF in psychiatric patients was assessed in a limited number of studies performed on subjects affected by depression [12], alcoholism [13], chronic psychiatric disorders $[14,15]$ and psychiatric outpatients [16].

Numerous studies in heterogeneous samples (both in the general population and in those suffering from different diseases) showed satisfactory psychometric properties of the WHOQOL-BREF tool [17]. The collaborative assessment of the WHOQOL-BREF scale in 15 cultural settings over several years confirmed good internal consistency (Cronbach's alpha coefficients for the domain scores ranged from 0.66 to 0.84 ) [18]. Also, assessment of the WHOQOL-BREF in some countries (Brazil, Iran, Spain) confirmed good internal consistency (Cronbach's alpha coefficient >0.7) [19]. Most of the validation studies revealed a four-factor structure of the WHOQOL-BREF scale [20]. Similar to studies in Australia [21] and China [22], one recent study showed that the WHOQOL-BREF tool is valid (four domains) and reliable (the Cronbach's alpha coefficient was over 0.7 for the questionnaire as a whole, and for all domains) for assessing quality of life among Saudi medical students [23]. To our knowledge, only few studies addressed the performance of WHOQOL-BREF on SCD patients and this research has never been conducted on patients suffering from this condition in Bahrain. Therefore, the aim of the present study was to assess the psychometric properties (Validity and Reliability Indicators) of the WHOQOL-BREF as a tool for the evaluation of Sickle Disease Patients in Bahrain. Thus, this user friendly tool might become a recommended asset to monitor and improve the QoL of these patients.

\section{Methods}

Study Design and Setting

A cross-sectional study design was used in this study. It enrolled (273) representative Sickle cell disease patients (above 21 years) from primary health-care centers (PHC) in Bahrain between July and August 2019. SCD patients enrolled in the study, voluntarily accepted to fill the WHOQOL-BREF during face to face interviews in the PHCs.

Study population 
The study population consisted of all Bahraini and Non-Bahraini, male and female, adult (21 years and above), who have confirmed Sickle Cell Disease (SCD) and who are followed-up at the SCD clinics at the primary health care in Bahrain.

Data Collection and Sampling Technique

The WHOQOL-BREF was administered for eligible volunteers at the PHCs during the month of June and July 2019. They represent a sample selected using systematic random sampling technique from the database of SCD patients available in the PHC. Patients were excluded if they have been diagnosed with any other health condition associated with chronic pain such as arthritis, chronic pain syndrome (CPS), low back pain (LBP), and/or psychiatric chronic diseases such as epilepsy, schizophrenia, and other mental illnesses. Vulnerable patients such as patients during the episode of pain crisis, pregnant women, or patients who refused or were unable to provide a valid informed consent were also excluded.

Instruments

The Arabic version of WHOQOL-BREF instrument was used in this study to ensure good understanding by patients. WHO Quality of Life Questionnaire contains a total of (26) items: items 3-26 represent four domains: Physical Health (7 items), Psychological Health (6 items), Social Relationships (3 items), and Environment (8 items). Two items ( 1 and 2 ) that are examined separately and refer to an individual's Overall perception of quality of life and an individual's Overall perception of general health. The answers for each item are given on a 1-5 Likert-type scale, where 1 denotes the least, and 5 is the highest agreement with a particular item. Items 3,4 , and 26 are negatively phrased and reversed during analysis. The mean score of items within each domain is used to calculate the domain score. Results on domains represent the sum of results of items. A higher sum of points represents a higher quality of life on a single domain.

\section{Statistical Analysis}

Psychometric properties of the WHOQOL-BREF were measured using the classical test theory. Reliability was measured by Cronbach's alpha coefficient to assess internal consistency. Pearson's correlation coefficient was used to investigate the association between scores of the scales' domains and perceptions of overall quality of life and overall general health. We conducted an exploratory factor analysis to evaluate construct validity using the Kaiser-Mayer-Olkin (KMO) index and Bartlett's sphericity test (BT). The principal component analysis relied on the extraction method, and the varimax rotation. A factor was considered as important if its eigenvalue exceeded 1.0. The goodness of fit was estimated by the $\chi^{2}$ test, the Root Mean Square Error of Approximation (RMSEA), the Comparative Fit Index (CFI) and Goodness of Fit Index Equation Model (SEM) and the confirmatory factor analysis (CFA). An RMSEA value $<0.08$ and a CFI value $>0.90$ indicated a good fit, also, for GFI a value $>0.90$ was an adequate model fit. A p-value less than 0.05 was considered as statistically significant for all tests. All statistical analyses were conducted using the Statistical Package for the Social Sciences (SPSS) version 27 (IBM, USA), and the Analysis of Moment Structures (AMOS) software version 23. 


\section{Results}

Basic characteristics of the study sample

The study sample was composed of 137 females (50.2\%) and 136 males (49.8\%). Regarding the education level, $157(57.7 \%)$ of the respondents had secondary level of education or below, while 115 $(42.3 \%)$ had BSc or high studies level of education. The mean age of the participants was $37.47 \pm 10.47$ years (range 20-70 years). The medical characteristics of SCD patients and quality of life level and determinants were detailed elsewhere (AlSaleh et al., accepted).

Psychometric and statistical methods

Reliability

Internal consistency reliability

The Reliability of the WHO quality of life as measured (Table 1) by Cronbach's alpha coefficient (value = 0.91) was satisfactory considering all score items, and when considered separately, for 'physical Health', 'psychological', 'social relationships, and 'environmental domains' $(0.83,0.72,0.67$ and 0.76 respectively).

Table 1

Reliability of WHOQOL-BREF Overall and domains' scores for SDC patients in Bahrain

\begin{tabular}{|llll|}
\hline Domain & No. of Items & Mean (SD) & Cronbach's a Coefficient \\
\hline Physical Health & 7 & $23.27(5.33)$ & 0.83 \\
\hline Psychological & 6 & $22.56(3.91)$ & 0.72 \\
\hline Social relationships & 3 & $11.97(2.47)$ & 0.67 \\
\hline Environmental & 8 & $28.54(5.31)$ & 0.76 \\
\hline Overall & 24 & $86.43(14.62)$ & 0.91 \\
\hline Validity & & & \\
\hline
\end{tabular}

Convergent validity

Table 2 shows the convergent validity for the WHO quality of life domains by using the Pearson's correlations Coefficients to detect correlation between the WHOQOL-BREF domains and with perceptions of "Overall Quality of Life", and "Overall General Health". Analysis indicated moderate correlation coefficients values for all domains, though statistically significant $(p<0.01)$. 
Table 2

Convergent Validity of the WHO quality of life domains: Correlation between scores of domains and Overall Quality of Life perception of Overall quality of life and overall general health

\begin{tabular}{|lll|}
\hline WHO Quality of Life Domains & Perception of QoL & Perception of Overall General Health \\
\hline Physical Health & $0.384 * \star$ & $0.417 * \star$ \\
\hline Psychological & $0.464 * \star$ & $0.492 * \star$ \\
\hline Social relationships & $0.346^{\star *}$ & $0.268 * \star$ \\
\hline Environmental & $0.424 * *$ & $0.391 * \star$ \\
\hline$* \star p<0.01$ & & \\
\hline
\end{tabular}

Exploratory factor analysis

Results showed the Kaiser-Meyer-Olkin (KMO) Measure to be 0.882 and the Bartlett's Test of Sphericity $(B T)$ to be chi-square $=1744.66(p<0.001)$, which confirms the suitability of the data in this study for factor analysis (Table 3). The exploratory factor analysis using principal components method revealed four factors with eigenvalues over one explaining $52 \%$ of cumulative variance in the 24 items. The rotated solution shows that each factor accounted for 10.34 to $15.1 \%$ of the total variance. The factor loadings of the 24 items onto the four factors are shown in Table 3. Factor 1 included all the (7) items of the physical domain and 3 items: one item (negative feeling), and two items of the environment domain (Leisure activity, and Health care), and it explained $15.08 \%$ of the rotated variance. Four items of the original psychology domain, and 3 items: one item (Energy of life) of the original physical health domain, and two items (Security, and Leisure activity) of the original environment domain, were included in factor 2 , and it explained $14.75 \%$ of the rotated variance. Factor 3 included all the (3) items of social relationships domain, and an item (satisfaction with self) of the original psychological domain, and it explained $11.83 \%$ of the rotated variance. Six items of the original environment domain, and an item (sexual activity) of the original social relationship's domain, were included in factor 4, and it explained $10.34 \%$ of the rotated variance. 
Table 3

Rotated Factor Matrix Solution for Factor analysis of 24 items

\begin{tabular}{|c|c|c|c|c|c|}
\hline \multirow[t]{2}{*}{ Item number } & \multirow[t]{2}{*}{ Item description } & \multicolumn{4}{|c|}{ Factor Loading } \\
\hline & & 1 & 2 & 3 & 4 \\
\hline & Physical Health & & & & \\
\hline 3 & Pain and discomfort & 0.782 & 0.108 & -0.093 & 0.236 \\
\hline 4 & Need for medical treatment & 0.720 & 0.099 & -0.111 & 0.141 \\
\hline 10 & Energy for life & 0.362 & 0.648 & 0.174 & 0.027 \\
\hline 15 & Mobility & 0.660 & 0.378 & 0.150 & 0.120 \\
\hline 16 & Sleep and rest & 0.583 & 0.272 & 0.443 & -0.040 \\
\hline 17 & Activities of daily living & 0.555 & 0.449 & 0.446 & 0.023 \\
\hline \multirow[t]{2}{*}{18} & Work capacity & 0.516 & 0.393 & 0.299 & 0.190 \\
\hline & Psychology & & & & \\
\hline 5 & Positive feeling & 0.262 & 0.643 & 0.021 & 0.298 \\
\hline 6 & Personal belief & 0.138 & 0.633 & 0.068 & 0.099 \\
\hline 7 & Concentration & 0.087 & 0.613 & 0.128 & 0.094 \\
\hline 11 & Body image & 0.159 & 0.555 & 0.257 & 0.098 \\
\hline 19 & Satisfaction with self & 0.232 & 0.272 & 0.524 & 0.381 \\
\hline \multirow[t]{2}{*}{26} & Negative feelings & 0.459 & 0.133 & 0.043 & 0.278 \\
\hline & Social relationships & & & & \\
\hline 20 & Personal relationships & 0.085 & 0.159 & 0.752 & 0.289 \\
\hline 21 & Sexual activity & 0.306 & 0.042 & 0.362 & 0.469 \\
\hline \multirow[t]{2}{*}{22} & Social support & -0.149 & 0.116 & 0.777 & 0.176 \\
\hline & Environment & & & & \\
\hline 8 & Security & -0.010 & 0.662 & -0.045 & 0.364 \\
\hline
\end{tabular}

Construct validity

The Confirmatory Factor Analysis (CFA) results showed that the four-domain structure of the WHOQOL-BREF produced an good fit to the data $\left(\chi^{2}=343.707, \mathrm{df}=232,\left(\chi^{2} / \mathrm{df}=1.481, \mathrm{p}<0.001\right)\right.$; CFI $=0.944 ; \mathrm{RMSEA}=0.042$ and $\mathrm{GFI}=0.907$. The factor load of each item with its respective domain ranged from 0.43 to 0.79 (figure. 1). These values were adequate, and the observed model showed good fit with the theoretical one revealing a good construct validity of the tool (Table 4). 


\begin{tabular}{|c|c|c|c|c|c|}
\hline \multirow[t]{2}{*}{ Item number } & \multirow[t]{2}{*}{ Item description } & \multicolumn{4}{|c|}{ Factor Loading } \\
\hline & & 1 & 2 & 3 & 4 \\
\hline 9 & Physical environment & -0.001 & 0.330 & 0.068 & 0.651 \\
\hline 12 & Financial support & 0.153 & 0.268 & 0.167 & 0.605 \\
\hline 13 & Accessibility of information & 0.286 & 0.250 & 0.149 & 0.366 \\
\hline 14 & Leisure activity & 0.362 & 0.503 & 0.240 & 0.158 \\
\hline 23 & Home environment & 0.165 & 0.149 & 0.146 & 0.646 \\
\hline 24 & Health care & 0.340 & 0.077 & 0.288 & 0.250 \\
\hline \multirow[t]{4}{*}{25} & Transport & 0.212 & -0.033 & 0.201 & 0.688 \\
\hline & Eigenvalues & 7.94 & 1.82 & 1.44 & 1.29 \\
\hline & Variance explained (\%) & 15.08 & 14.75 & 11.83 & 10.34 \\
\hline & Cumulative variance (\%) & 15.08 & 29.83 & 41.66 & 52.00 \\
\hline \multicolumn{6}{|c|}{ Construct validity } \\
\hline \multicolumn{6}{|c|}{$\begin{array}{l}\text { The Confirmatory Factor Analysis }(\mathrm{CFA}) \text { results showed that the four-domain structure of the } \\
\text { WHOQOL-BREF produced an good fit to the data }\left(\chi^{2}=343.707, \mathrm{df}=232,\left(\chi^{2} / \mathrm{df}=1.481, \mathrm{p}<0.001\right) ; \mathrm{CFI}\right. \\
=0.944 ; \mathrm{RMSEA}=0.042 \text { and } \mathrm{GFI}=0.907 \text {. The factor load of each item with its respective domain } \\
\text { ranged from } 0.43 \text { to } 0.79 \text { (figure. } 1) \text {. These values were adequate, and the observed model showed } \\
\text { good fit with the theoretical one revealing a good construct validity of the tool (Table } 4 \text { ). }\end{array}$} \\
\hline
\end{tabular}

Table 4

Fitness statistics for the four factor-analytic models of the WHOQOL Questionnaire

\begin{tabular}{|lllllll|}
\hline Model & $\chi^{2}$ & df & $\chi^{2} / \mathbf{d f}$ & GFI & CFI & RMSEA (90\% C.I) \\
\hline Original model & 581.203 & 246 & 2.363 & 0.850 & 0.830 & $0.07(0.04-0.08)$ \\
\hline Modified model & 343.707 & 232 & 1.481 & 0.907 & 0.944 & $0.042(0.01-0.05)$ \\
\hline $\begin{array}{l}\text { X2: chi-square; df: degree of freedom; CFI: Comparative Fit index; GFI: Goodness of Fit Index; RMSEA } \\
\text { = Root Mean Square Error of Approximation. }\end{array}$ \\
\hline
\end{tabular}

\section{Discussion}

The aim of this study was to examine the validity and reliability of the WHOQOL-BREF in the evaluation of the quality of life using a representative sample of (273) Sickle Disease patients in Bahrain. The results of our study confirmed the validity and reliability of the WHOQOL - BREF as a tool for the measurement of the quality of life of SCD patients in Bahrain. Indeed, Cronbach's alpha coefficients for all domains (physical health, psychological, social relationships, and environmental) were $0.83,0.72$, 0.67 , and 0.76 , respectively. Except for the social relationship's domain, the coefficients of the three 
domains were above acceptable value 0.7 , which indicates a good internal consistency of this tool [24]. A lower alpha coefficient of the social relationship's domain ranging from 0.58 and 0.66 was also reported in other studies [6, 22, 25]. Similar findings were reported in multiple quality of life studies in Pakistan[19], Saudi Arabia [23]. Lower alpha values for the social domain are most probably due to the unrelated and limited number of three items in this domain. Failing to use the recommended minimum of four items in assessment of internal consistency in this domain to obtain at least 0.70 might be the reason for this result [26].

Regarding convergent validity, our findings indicated that all values of the correlation coefficients were statistically significant. Also, the current study confirmed that the psychological domain has highly contributed for both overall quality of life, and perception of overall general health. This result disagrees with previously reported findings. Noticeably, in a study of patients with physical impairments in Korea, the psychological domain was the main contributor of overall quality of life, whereas the physical domain is highly associated with general health[27]. A study of Polish respondents has also shown that the psychological domain has the strongest contribution for overall quality of life, whereas the physical domain was the contributor of general health followed by the psychological domain[28]. However, the physical domain was found to be a strong contributor for both overall quality of life and general health in a study conducted in India among people with type 2 diabetes [29]. These discrepancies could be explained by the nature and specificities of the related health problems.

Regarding the validity, the results of exploratory factor analysis (EFA) showed the presence of four main factors with an eigen value greater than 1 , explaining $52 \%$ of variance. These findings corroborate those shown in the international study (WHOQOL-BREF field trial), where the four factors explained $53 \%$ of variance[10]. However, they are slightly lower than those reported from a study conducted in China, where the four domains cumulative contribution was 69.3\% [22]. The EFA results showed that factor 1 is composed of similar items with the physical health domain. The results also indicated that six items are loaded on another domain different from their original one. Among these items, those related to satisfaction and self and negative feelings were loaded on the social relationships and physical health domains, respectively. While items of sexual activity related to social relationship's domain was loaded on the environment domain. Also, items security and leisure activity of the environment domain were loaded on the psychological domain. On the other hand, item health care of the environment domain was loaded on the physical health domain. It is also important to note that the item energy for life from the original physical health domain was loaded on the psychological domain. Cross-loading of items with other domains in the context of the evaluation of the quality of life of traumatic spinal cord injury using WHOQOL-BREF was also reported elsewhere [30].

The confirmatory factor analysis (CFA) provided an acceptable fit to a four-factor model in the sickle disease patient's sample. Regarding the CFA, the original model has shown indices of $(\mathrm{CFI}=0.830, \mathrm{GFI}=$ 0.85 and RMSEA $=0.07$ ), which indicates that the domains in model did not fit for the sickle disease patients. However, the model gained acceptable goodness of fit ( $\mathrm{CFI}=0.944, \mathrm{GFI}=0.907$ and RMESA $=$ 0.042) after three pairs of error variance were allowed to covary (i.e., item 19 (psychological) and item 20 
(Social relationship); item 5 (psychological) and item 14 (Environment); item 16 (physical health) and item 24 (Environment); and after three pairs of items were allowed to cross-load on other domains: physical health domain and item 14 (Environment); social relationship domain and item 19 (psychological); psychological domain and item 8 (social relationship). Overall, the four-domain model fitted reasonably well the model in the sickle disease patient's sample. This is in line with the findings of different studies which used WHOQOL-BREF. In a validation study conducted among substance users in northern Taiwan, the modified model has shown acceptable goodness of fit of indices $\mathrm{CFI}=0.92, \mathrm{NNFI}=$ 0.91 , and RMSEA $=0.06$ [31]. In a study conducted among older people in Taiwan, the CFI was increased to 0.90 from 0.85 when three pairs of error variances could covary and two items were allowed to crossload on other domains [32]. A study which was conducted in Ethiopia among patients with diagnosed type 2 diabetes has found the model gained acceptable goodness of fit (CFI $=0.94$, NNIF $=.95$, and RMSEA $=0.05$ ), after removing 2 items (item 7 from psychological domain, and item 23 from environmental domain), and after item 9 (Physical environment) from environment domain are allowed to cross-load on physical health and social domains, respectively[33].

Strengths and limitations

This is the first study to measure the psychometric properties of the WHOQOL-BREF for SCD patients in Bahrain, and this area has never been addressed previously although SCD is highly prevalent. The study was limited by the retrospective character of the cross-sectional design which might lead to a recall bias as well as the impossibility to examine the instrument's test-retest reliability. Young patients and the most severe ones were not included in the present study for ethical and feasibility reasons which might compromise the generalizability of findings. Future prospective studies using larger samples and enrolling patients from secondary care are required to have a more valid and representative evaluation of the tool.

\section{Conclusion}

This study confirmed the usefulness of the WHOQOL-BREF in the measurement of the quality of life of SCD patients using a representative sample from Bahrain. It revealed good psychometric properties (Reliability \& Validity) and should be recommended for future use in this health condition impact assessment on patients. However, more studies are required to improve the reliability results in the 'social relationship' domain. This gap might be addressed using a qualitative study design approach which is favored because of its inductive nature in generating items within constructs. Quantitative prospective studies on larger samples of patients including the whole severity spectrum of SCD are required.

\section{Abbreviations}

QoL

Quality of life

SCD 
Sickle cell disease

PHCs

Primary Health-Care centers

CPS

Chronic Pain Syndrome

LBP

Low Back Pain

EFA

Exploratory Factor Analysis

CFA

Confirmatory Factor Analysis

$\mathrm{KMO}$

Kaiser-Mayer-Olkin

$\mathrm{CFI}$

Comparative Fit Index

GFI

Goodness of Fit Index

RMSEA

Root Mean Square Error of Approximation

BT

Bartlett's sphericity test

AGU

Arabian Gulf University

CMMS

College of Medicine and Medical Sciences

$x^{2}$

chi-square

df

degree of freedom

\section{Declarations}

\section{Ethics declarations}

This study was approved by the Ethical Committee in the College of Medicine and Medical Sciences, Arabian Gulf University (approval number: E002-pi-4/19) and the ethical committee of the ministry of health in the kingdom of Bahrain (approval number: AURS/325/2019). All participants provided informed written consent before participation.

Consent for publication 
All authors have read the manuscript and agreed to all its contents. All authors gave their consent for publication.

\section{Availability of data and materials}

The datasets used during the current study are available upon request from the corresponding author.

\section{Competing interests}

The authors declare that they have no competing interests.

\section{Funding}

This research received no external funding.

\section{Author's Contributions}

$A A$ and $A S$ conceived and designed the study; $A E, A Q, F A, M S$ and $M S$ performed the research process and collected the data; $A A$ performed the statistical analyses; $A A, A S, S C$ and $M G$ wrote the original draft of the manuscript; AA, AS and SC prepared the figures and tables; AS, HA, LA, NF, MY and AA edited and revised the manuscript; $A A, A S$ was the project manager; $A A, A S$ and $M K$ approved the final version of the manuscript.

\section{Acknowledgements}

The authors would like to thank the Sickle Disease Patient in Bahrain for their participation in this study.

\section{References}

1. Piel FB, Steinberg MH, Rees DC. Sickle cell disease. New England Journal of Medicine. 2017;376(16):1561-73.

2. Brandow AM, Zappia KJ, Stucky CL. Sickle cell disease: a natural model of acute and chronic pain. Pain. 2017;158(Suppl 1):S79.

3. El-Hazmi MA, Al-Hazmi AM, Warsy AS. Sickle cell disease in Middle East Arab countries. The Indian journal of medical research. 2011;134(5):597.

4. Thein MS, Thein SL. World Sickle Cell Day 2016: A time for appraisal. The Indian journal of medical research. 2016;143(6):678.

5. Merrill RM. Introduction to epidemiology: Jones \& Bartlett Publishers; 2015.

6. Katschnig H. How useful is the concept of quality of life in psychiatry? Current opinion in Psychiatry. 1997;10(5):337-45. 
7. Orley J, Saxena S, Herrman H. Quality of life and mental illness: reflections from the perspective of the WHOQOL. The British Journal of Psychiatry. 1998;172(4):291-3.

8. Barry MM, CROSEY C. Quality of Life as an Evaluative Measure in Assessing the Impact of Community Care on People with. British Journal of Psychiatry. 1996;168:210-6.

9. Saxena S, Quinn K, Sharan P, Naresh B, Yuantao H, Power M. Cross-linguistic equivalence of WHOQOL100: a study from North India. Quality of Life Research. 2005;14(3):891-7.

10. Skevington SM, Lotfy M, O'Connell KA. The World Health Organization's WHOQOL-BREF quality of life assessment: psychometric properties and results of the international field trial. A report from the WHOQOL group. Quality of life Research. 2004;13(2):299-310.

11. De Girolamo G, Becchi MA, Coppa F, De Leo D, Neri G, Rucci P. Salute e qualità della vita. 2001.

12. Berlim MT, Pavanello DP, Caldieraro MA, Fleck MP. Reliability and validity of the WHOQOL BREF in a sample of Brazilian outpatients with major depression. Quality of life research. 2005;14(2):561-4.

13. Da Silva Lima AB, Fleck M, Pechansky F, De Boni R, Sukop P. Psychometric properties of the World Health Organization quality of life instrument (WHOQoL-BREF) in alcoholic males: a pilot study. Quality of Life Research. 2005;14(2):473-8.

14. Herrman H, Patrick DL, Diehr P, Martin ML, Fleck M, Simon GE, et al. Longitudinal investigation of depression outcomes in primary care in six countries: the LIDO study. Functional status, health service use and treatment of people with depressive symptoms. Psychological medicine. 2002;32(5):889-902.

15. Van de Willige G, Wiersma D, Nienhuis FJ, Jenner JA. Changes in quality of life in chronic psychiatric patients: a comparison between EuroQol (EQ-5D) and WHOQoL. Quality of Life Research. 2005;14(2):44151.

16. Trompenaars FJ, Masthoff ED, Van Heck GL, Hodiamont PP, De Vries J. Content validity, construct validity, and reliability of the WHOQOL-Bref in a population of Dutch adult psychiatric outpatients. Quality of Life Research. 2005;14(1):151-60.

17. Bulamu NB, Kaambwa B, Ratcliffe J. A systematic review of instruments for measuring outcomes in economic evaluation within aged care. Health and quality of life outcomes. 2015;13(1):1-23.

18. Power M, Bullinger M, Harper A. The World Health Organization Quality of Life Group: The World Health Organization WHOQOL-100: Tests of the universality of quality of life in 15 different cultural groups worldwide. Health Psychology. 1999;5:495-505.

19.Lodhi FS, Raza O, Montazeri A, Nedjat S, Yaseri M, Holakouie-Naieni K. Psychometric properties of the Urdu version of the World Health Organization's quality of life questionnaire (WHOQOL-BREF). Medical journal of the Islamic Republic of Iran. 2017;31:129. 
20.Vahedi S. World Health Organization Quality-of-Life Scale (WHOQOL-BREF): analyses of their item response theory properties based on the graded responses model. Iranian journal of psychiatry. 2010;5(4):140.

21.Krägeloh C, Henning M, Hawken S, Zhao Y, Shepherd D, Billington R. Validation of the WHOQOL-BREF quality of life questionnaire for use with medical students. 2011.

22.Zhang Y, Qu B, Lun S, Wang D, Guo Y, Liu J. Quality of life of medical students in China: a study using the WHOQOL-BREF. PloS one. 2012;7(11):e49714.

23.Malibary H, Zagzoog MM, Banjari MA, Bamashmous RO, Omer AR. Quality of Life (QoL) among medical students in Saudi Arabia: a study using the WHOQOL-BREF instrument. BMC medical education. 2019;19(1):1-6.

24. Privitera GJ. Statistics for the behavioral sciences: Sage; 2011.

25. Chung W-S, Lan Y-L, Yang M-C. Psychometric testing of the short version of the world health organization quality of life (WHOQOL-BREF) questionnaire among pulmonary tuberculosis patients in Taiwan. BMC public health. 2012;12(1):1-10.

26.Cohen J. Statiscal Power Analysis for the Behavioral Sciences, Secon. New Jersey: La Wrence Erlabaum Associates, Publishers; 1988.

27. Kim WH, Hahn SJ, Im HJ, Yang KS. Reliability and validity of the Korean World Health Organization Quality of Life (WHOQOL)-BREF in people with physical impairments. Annals of rehabilitation medicine. 2013;37(4):488.

28. Jaracz K, Kalfoss M, Górna K, Bączyk G. Quality of life in Polish respondents: psychometric properties of the Polish WHOQOL-Bref. Scandinavian Journal of Caring Sciences. 2006;20(3):251-60.

29. Sreedevi A, Cherkil S, Kuttikattu DS, Kamalamma L, Oldenburg B. Validation of WHOQOL-BREF in Malayalam and determinants of quality of life among people with type 2 diabetes in Kerala, India. Asia Pacific Journal of Public Health. 2016;28(1_suppl):62S-9S.

30. Jang $\mathrm{Y}$, Hsieh C-L, Wang Y-H, Wu Y-H. A validity study of the WHOQOL-BREF assessment in persons with traumatic spinal cord injury. Archives of Physical Medicine and Rehabilitation. 2004;85(11):1890-5.

31. Fu TS-T, Tuan Y-C, Yen M-Y, Wu W-H, Huang C-W, Chen W-T, et al. Psychometric properties of the World Health Organization quality of life assessment-brief in methadone patients: a validation study in northern Taiwan. Harm Reduction Journal. 2013;10(1):1-8.

32. Hwang H-F, Liang W-M, Chiu Y-N, Lin M-R. Suitability of the WHOQOL-BREF for community-dwelling older people in Taiwan. Age and ageing. 2003;32(6):593-600. 
33. Reba K, Birhane BW, Gutema H. Validity and reliability of the Amharic version of the World Health Organization's quality of life questionnaire (WHOQOL-BREF) in patients with diagnosed type 2 diabetes in Felege Hiwot referral hospital, Ethiopia. Journal of diabetes research. 2019;2019.

\section{Figures}

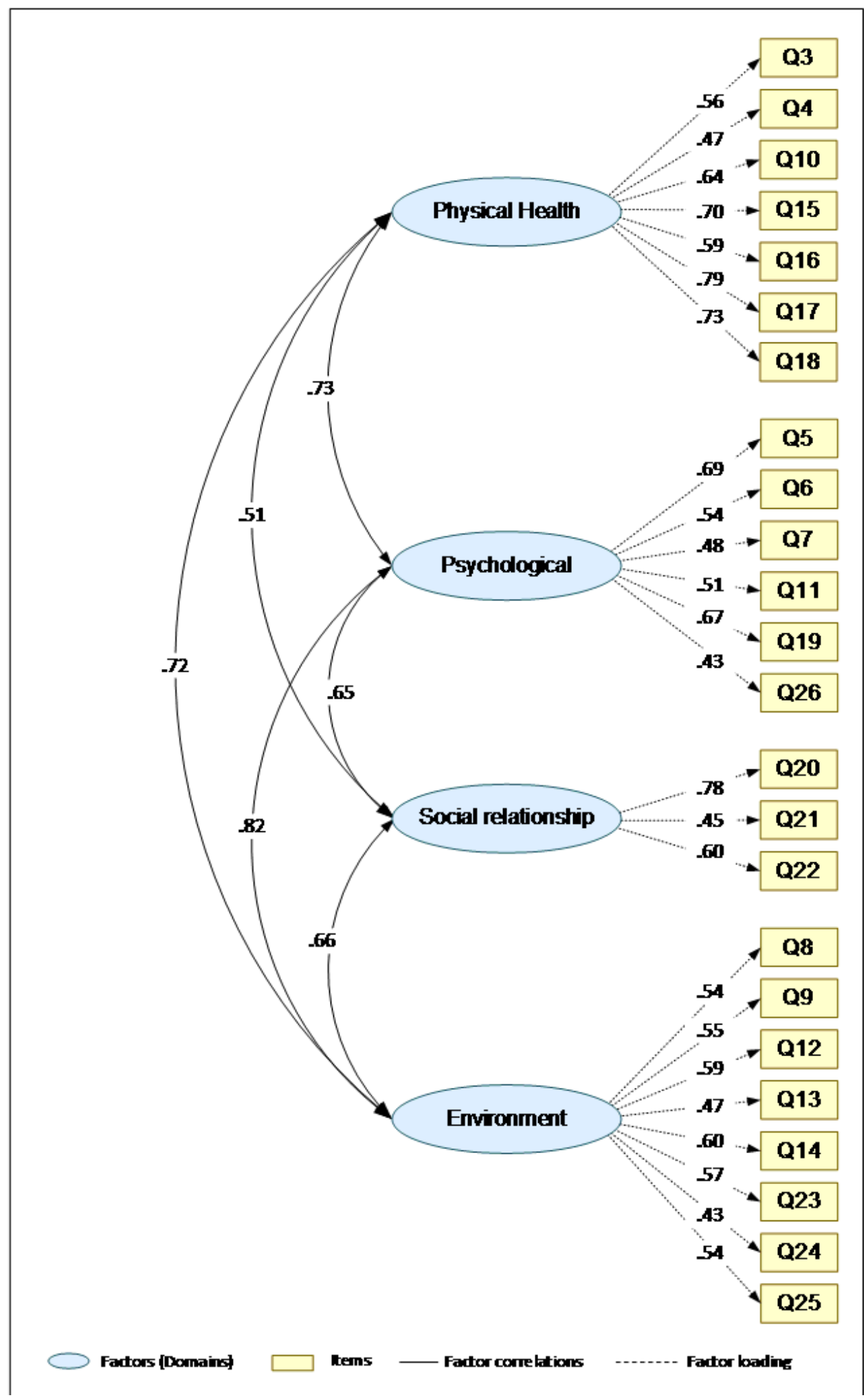

Figure 1 
The structure of the WHOQOL-BREF based on confirmatory factor analysis. 